\title{
Conversational Intelligence Analysis
}

\author{
Alice Toniolo \\ Dept. of Computing Science \\ University of Aberdeen, UK
}

\author{
Timothy J. Norman \\ Dept. of Computing Science \\ University of Aberdeen, UK
}

\author{
Dave Braines \\ Emerging Tech. Services, \\ IBM United Kingdom Ltd \\ Hursley Park, Winchester, UK \\ Paul Sullivan \\ INTELPOINT Incorporated \\ Pennsylvania, USA
}

\author{
Alun D. Preece, Will Webberley \\ School of Computer Science \\ and Informatics \\ Cardiff University, UK
Timothy Dropps
Honeywell \\ USA
}

\begin{abstract}
Social networks foster the development of social sensing to gather data about situations in the environment. Making sense of this information is, however, a challenge because the process is not linear and additional sensed information may be needed to better understand a situation. In this paper we explore how two complementary technologies, Moira and CISpaces, operate in unison to support collaboration among human-agent teams to iteratively gather and analyse information to improve situational awareness. The integrated system is developed for supporting intelligence analysis in a coalition environment. Moira is a conversational interface for information gathering, querying and evidence aggregation that supports cooperative data-driven analytics via Controlled Natural Language. CISpaces supports collaborative sensemaking among analysts via argumentation-based evidential reasoning to guide the identification of plausible hypotheses, including reasoning about provenance to explore credibility. In concert, these components enable teams of analysts to collaborate in constructing structured hypotheses with machine-based systems and external collaborators.
\end{abstract}

\section{CCS Concepts}

-Theory of computation $\rightarrow$ Automated reasoning; -Human-centered computing $\rightarrow$ Computer supported cooperative work; Natural language interfaces;

\section{Keywords}

Argumentation; Controlled Natural Language; Provenance; Intelligence Analysis

\section{INTRODUCTION}

The rapid increase of social network development has created greater opportunities to leverage social sensing as methods to collect data about the environment. In social sensing, people act as sensors, share information within a network or

ACM acknowledges that this contribution was authored or co-authored by an employee, or contractor of the national government. As such, the Government retains a nonexclusive, royalty-free right to publish or reproduce this article, or to allow others to do so, for Government purposes only. Permission to make digital or hard copies for personal or classroom use is granted. Copies must bear this notice and the full citation on the first page. Copyrights for components of this work owned by others than ACM must be honored. To copy otherwise, distribute, republish, or post, requires prior specific permission and/or a fee. Request permissions from permissions@acm.org.

ICDCN '16, January 04-07, 2016, Singapore, Singapore

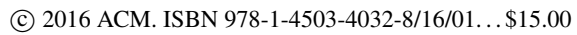

DOI: http://dx.doi.org/10.1145/2833312.2849568 respond to data or opinion requests [1]. In order to make sense of a situation, however, a consumer (e.g., analyst or decision maker) would need to analyse and link this information to other contextual evidence, gathered from soft or hard sources, to identify explanations of the environment. This often happens in intelligence and policy analysis, emergency response, and other scenarios where the consumer must draw conclusions over situations to inform actions. Making sense of information may be a social activity, where many contributors help identify different plausible hypotheses. The process is iterative: the consumer may source further information from social networks during the analysis particularly when there are conflicting view points; further information may also be reported by social sensors highlighting new perspectives. Additionally, the credibility of explanation must be considered in the context of the origins of the underpinning information (i.e., provenance). Existing systems, however, mainly focus on either ends of the process to: sense data and perform data analytic tasks (e.g. $[6,10])$ or present explanations (e.g. [6]). Here we address the problem of how to support the iterations between sensing and sensemaking from information to hypotheses and back, to develop awareness of highly evolving situations.

In this paper, we explore the integration of two complementary agent-based technologies: Moira (Mobile Intelligence Reporting Agent [9]) and CISpaces (Collaborative Intelligence Spaces [12]). We show how these support humanagent teams in gathering and analysing information for situation awareness [2]. Conversational sensing and argumentation-based reasoning underpin the system to enable collaborative intelligence analysis. CISpaces facilitates the reasoning process of an analyst team by structuring and sharing analyses of conflicting information through argumentation techniques and maintains records of the provenance information [12]. The sensing of information from hard or soft sensors must, however, be processed to be understandable by human analysts. Moira is a Controlled Natural Language (CNL) conversational agent that captures sensed information by supporting queries and intelligence provision from a wide set of different sources, such as physical sensors and information systems, social media and human sources [9].

The complementary support that CISpaces and Moira offers can strengthen the capability of analysts to collaborate with human and agent-based support services. By facilitating collaborative analysis and conversation with agents, our integrated system enables a more complete and robust delivery of analytical products for improving situation awareness that can be exploited in different domains. 


\section{INTELLIGENCE ANALYSIS}

Intelligence analysis is the process of elaborating information to determine plausible hypotheses for situations and events and identify likely threats or opportunities in a timely manner [3]. The phases of analysis range from gathering field intelligence, hypothesising causes of events to postevent forensic reconstruction. Among the many definitions of the intelligence analysis process, Pirolli and Card [7] conceptualise this as two interleaving loops (referred to as $\mathrm{P} \& \mathrm{C}$ model): foraging for information, where analysts gather information relevant to the situation and sensemaking where hypotheses are generated and tested against the evidence built upon information. Reports generated from plausible hypotheses are sent to decision makers who act upon this.

Similarly, the analysis of information from social network sensing follows the $\mathrm{P} \& \mathrm{C}$ model and can be utilised by decision makers in a variety of contexts, such as policy analysis, emergency response, journalism, etc. CISpaces and Moira support collaboration in the foraging and sensemaking loops: collaboration increases capabilities and allows different viewpoints to be confronted and reflected upon, thereby reducing risk of cognitive biases. Collaboration, however, increases the cognitive load on analysts in dealing with large amount of conflicting information and in validating the different perspectives. Our objective is to provide support to analysts to increase the reliability of their analysis by leveraging the work of many human/agent contributors.

\subsection{Foraging and Sensemaking in Moira}

Moira is a Controlled Natural Language (CNL) conversational agent, that uses a conversational model based on the syntax and semantics of the ITA Controlled English (CE) [8]. CE is a subset of natural English language directly processable by machine agents with no ambiguity; CE defines syntax and structure, and allows complex semantics based on First Order Predicate Logic to be expressed [5].

In our previous research into Conversational Sensemaking [10] we applied the Moira human-machine conversational capability showing how the use of a common human-machine language like $\mathrm{CE}$ is able to underpin both the $\mathrm{P} \& \mathrm{C}$ foraging and sensemaking loops. By applying this methodology to existing social network sources such as Twitter we are able to apply a simple "Bag of words" natural language processing technique to identify the contextual meaning behind messages from users in an area of interest or on a given topic. This approach works by taking a previously defined CE model of the domain of interest and attempting to map the existing user messages onto this domain, identifying references to key entities of interest, or assertions of concepts within the domain of interest (such as crowds, riots, damage etc). In addition to simply "absorbing" existing social media, this exact approach also underpins the conversational mechanism that can be used to request and subsequently process such information from human agents in the field.

All of the incoming "field intelligence" constitutes raw data that may be relevant to the current situation and in the terms of $\mathrm{P} \& \mathrm{C}$ constitutes information in the "shoebox" that has been gathered during the foraging loop. The sensemaking loop uses this raw data, takes into account hypotheses and additional relevant contextual information and attempts to "increase the schematisation" of the data from the shoebox to better position it to inform situation awareness and decision making. A key observation in the application of

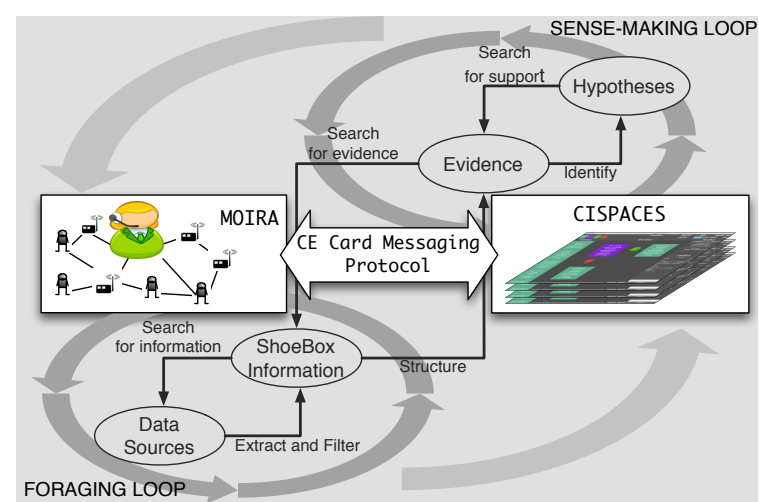

Figure 1: Pirolli and Card adapted model

Controlled Natural Language techniques to the P\&C approach is that the "schematisation" is driven down into the foraging loop and the shoebox, enabling machine support for processing of structured data even at that very early stage.

In order to enable sensemaking, Moira employs a humanmachine conversational approach engaging analysts and other members and services of the coalition in a natural language conversation. Moira uses the CE Cards messaging protocol [8] to manage conversations between agents using both natural language and Controlled Natural Language. The CNL employed by Moira is designed to be both easily machine processable and easily human-readable with some additional effort required to be able to write it. Thus, Moira facilitates the human-agent interaction via messages that flow from easy-to-write natural language from the human users to easy-to-read CNL by the machine agents, and back again. The information gathered as a result of this conversation among agents offers additional raw intelligence information into the system, and can also surface existing relevant raw information to the end users via conversational question asking, if the policies allow access to that information.

\subsection{Foraging and Sensemaking in CISpaces}

The development of CISpaces, proposed in our previous research [12], was driven by the need of employing agents to support reasoning with different types of evidence: information coming from different intelligence sources, responses to structured information via crowdsourcing and provenance of information. The CISpaces toolkit is based upon a graphical representation of arguments structured and analysed through argumentation-based reasoning and argument schemes to support the identification of plausible hypotheses [13]. While offering some support to foraging for information, CISpaces is focussed on enabling collaboration at the core of the sensemaking process, where analysts link information, reason about corroborating or contradicting evidence and share partial analyses with other collaborators. In contrast with existing systems (e.g. [6]), CISpaces aims at facilitating and improving reasoning via argumentation techniques and provides semi-automatic support for interpreting information, provenance and crowdsourced data to generate well-supported and credible explanations of evidence.

\subsection{Challenges of an Integrated System}

The integration of Moira and CISpaces systems can effectively enable both $\mathrm{P} \& \mathrm{C}$ foraging and sensemaking iterative loops as shown in Figure 1. In the integrated system, the 


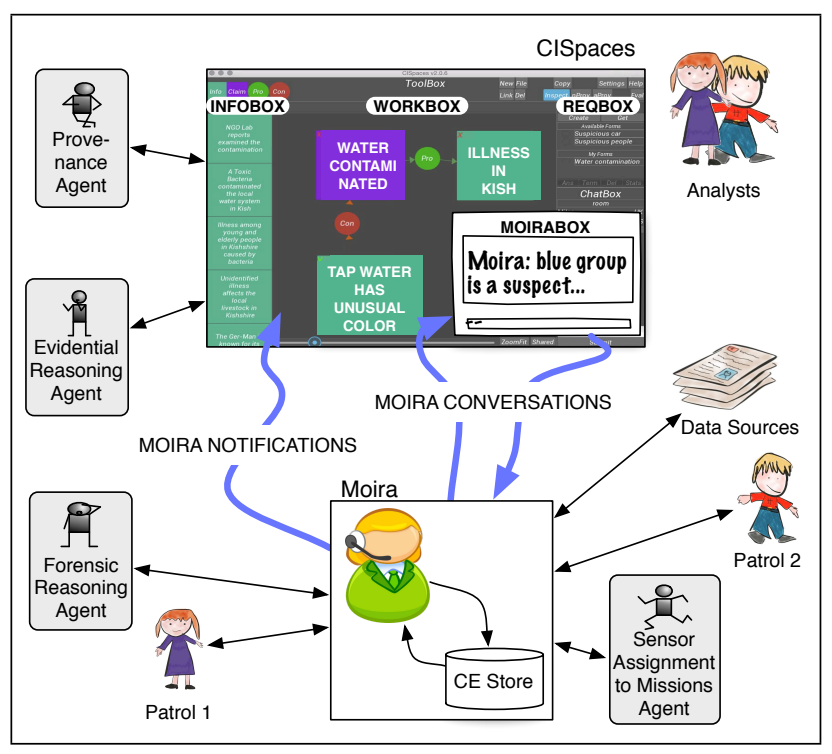

Figure 2: Moira and CISpaces

sensemaking part of the process of Moira shifts more towards the CISpaces component, and towards an active human-led process, but there is still a role for appropriate machine agents to assist the human users with any automatable processes that could otherwise lead to information overload.

The transition between foraging and sensemaking in the $\mathrm{P} \& \mathrm{C}$ process is leveraged by evidence collection. This is a crucial point in the analysis because new evidence requirements must be formulated and sent to relevant collectors. Results need to be analysed and sent back in a format that is understandable and makes sense for the human analysts. The highly time-stressed environment of intelligence operations increases analysts' cognitive burden. In our integrated system, this transition is enabled by the CE Cards messaging protocol provided by Moira. New evidence requirements lead the analysts working in CISpaces to engage in a conversation with Moira, enabling consultation via natural language with both human and machine agents via a simple standard interface. Moira may also send incoming notifications to the analysts via CISpaces, again from either machine inferences or key local knowledge provided by human agents in the field. The uniform structure of links and inferences amongst information is employed by Moira to import and easily integrate relevant intelligence from agentbased services and contributors within the coalition. Hence, the CISpaces foraging process fully relies on Moira, which provides a more suitable and comprehensive platform for human/machine reports, while both systems are responsible for recording provenance data.

\section{CISPACES AND MOIRA AGENTS}

Here we describe the underpinning agent-based techniques that permit Moira and CISpaces to provide effective support to analysts. An overview is shown in Figure 2.

\subsection{Moira Agent}

Moira provides a mobile app for field patrols allowing them to provide information (e.g. event reports) and receive updates (e.g. BOLO — be on the lookout — bulletins) [9]. Moira mediates the conversation between field patrols, ana-

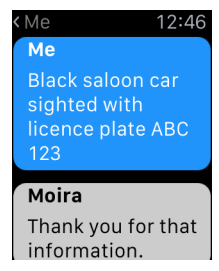

Figure 3: Moira interaction on a smart watch

lysts and a variety of human/agent services, to gather intelligence and process requirements.

A common mode of interaction with Moira is a confirmatory dialogue that starts with the user providing some input in natural language (e.g. via keyboard or speech) and the agent performing natural language processing to interpret the input into CNL form. For example, a user might report, "Black saloon car sighted, with licence plate ABC 123 ", which Moira could process as the following CNL:

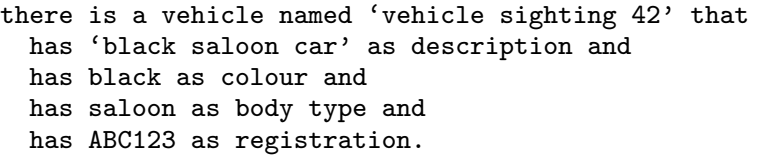

The user may then confirm whether Moira's interpretation is acceptable (the human readability of CNL is important in enabling this to happen) or modify it. Once confirmed, the new information can be processed and combined with other knowledge. For example, in this case, some other known associations about that particular vehicle may trigger the issuing of a BOLO message via Moira.

Use of the Moira conversational interaction allows users to request "rationale" for received information, by asking why, with Moira responding with any information relating to the premises that led to any conclusions in the original statement (e.g. the rationale for a BOLO). The CE cards protocol also permits Moira to call upon other services such as the SAM (Sensor Assignment to Missions) agent for tasking ISR (Intelligence, Surveillance and Reconnaissance) assets.

Interactions via Moira are governed by policies so that, for example, only particular users may be permitted to receive rationale, or initiate conversations with agents capable of tasking sensing assets. In certain cases, rather than providing a CNL interpretation of the user's input for confirmation, Moira may simply acknowledge receipt, to avoid revealing any aspects of how the received information had been modelled (i.e., what it interprets as being important about the user's input). Current implementations of Moira run on a range of mobile devices, including tablets, smart watches, and smartphones. An example smart watch screenshot of a policy-governed interaction is shown in Figure 3.

\subsection{CISpaces Interface}

The CISpaces interface provides individual and shared spaces for intelligence analysis as well as access to Moira. The CISpaces interface includes an InfoBox, where information is streamed from Moira, and a WorkBox, the space for hypotheses construction and sensemaking of information. Collaboration is supported in CISpaces via a shared WorkBox, which analysts can switch to when intending to share partial analyses. The evidential reasoning agent facilitates the reasoning process by guiding the construction of hypotheses as graphical argument maps where users can draw 
supports, attacks or preferences between nodes. Moreover, a provenance agent employs rigorous methods of recording and interrogating the origins of information. New intelligence requirements are initiated by the users via the MoiraBox, a dedicated chat box from which analysts can engage with Moira and import newly processed intelligence into their space of analysis.

\subsection{Evidential Reasoning Agent}

The WorkBox provides a space of analysis where inferences between information and claims are structured. The evidential reasoning agent maps this graph of inferences to an argumentation framework [12], where "Pro" (green) links form arguments and "Con" (red) links represent attacks. A new feature is added to CISpaces: analysts can add preference (purple) nodes "Pref" indicating that a node is preferred to another and the framework is extended to handle conflicting preferences. The agent interprets annotation of reasons for "Pro" links as argument schemes. Schemes are patterns of reasoning composed by premises and conclusions [13]. Premises are formed by intelligence elements as facts, entities and events, and conclusions are tentatively drawn by discovering causal or associative relations between these elements. A scheme may be expressed as:

- $n_{1}$ : Given a general relation, a previous case, or some sign of a situation that may happen

- $n_{2}$ : Some events took place, or some properties of entities held $\Rightarrow n_{3}$ : Then, some other events may plausibly have taken place, or some other properties of entities hold

Each scheme is associated with critical questions (CQs) suggested by the agent to challenge and avoid biases, thereby embodying key techniques within the intelligence analysis process [3]. CQs are critiques for premises or conclusions, such as "Does this fact hold?"; other CQs may challenge the relationships between elements, such as "Is it the case that generally if an event takes place, a related event follows?".

The agent provides support to analysts to identify plausible hypotheses via an argumentation framework extended from [12]. The framework used is composed by a knowledgebase and a set of defeasible rules underpinned by a propositional logic language. Arguments are constructed as a chain of inferences from the knowledge-base and may lead to assert inconsistent/conflicting conclusions. Preferences are part of the language and may be formed to resolve conflicts under certain conditions. This is mapped to an abstract framework where arguments and attacks between arguments are defined. On the basis of this structure, an argumentation semantics is used to extract sets of arguments that are acceptable and considered a collectively reasonable position for a controversial standpoint that an agent can agree upon.

In CISpaces, the argumentation framework is extracted from the current WorkBox view, mapped to a set of nodes $N=\left\{n_{1}, n_{2}, n_{3}, \ldots\right\}$, and evaluated. The evaluation presents to the analyst $k$ options representing alternative hypotheses. For each option in $k, N$ is partitioned in subsets of nodes: supported indicated with ' $V$ ' representing a node defended against its attackers; unsupported indicated with ' $X$ '; and undecided, referred to as '?', representing a node that has insufficient grounds to be either supported or unsupported.

\subsection{Provenance Agent}

For effective collaborative analysis, the provenance of both source information and previous analyses must be considered. Provenance data helps analysts to understand how a

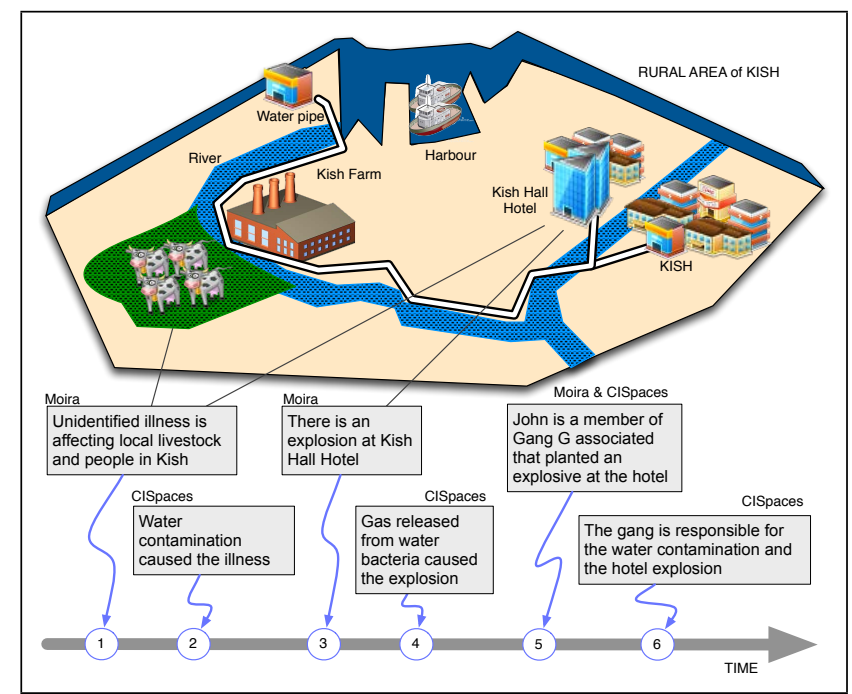

Figure 4: Scenario and analysis steps example

piece of information was handled and why. Provenance is recorded using the PROV-DM standard [4] that represents activities, entities and actors involved in the generation of an entity. The provenance agent is employed to elaborate this data and identify influential provenance elements, extracted as patterns. The analyst may select a relevant pattern, and the agent constructs a "Pro" link instantiating a scheme that uses the pattern as a premise to warrant the credibility of the information in the analysis space [11]. When a conflict between claims arises, an argument scheme helps analysts to select those more preferred. The scheme expresses a preference between two nodes of information in order to enable the resolution of conflicts according to different criteria [11]. Some information may be more trustworthy when issued by sources that are more likely to report truthfully; reliable if it is elaborated through processes known to perform well; timely when it is more recent; and accurate if it is closer to the primary sources than other conflicting information.

Recording provenance data will help the audit trail, showing what evidence underpinning hypotheses was available at the time of analysis to improve future analyses and enable better understanding of the "point in time" view that was applicable at the time of analysis.

\section{SCENARIO AND EXAMPLE}

In this section, we present our motivating scenario and a 'step-by-step' example of the support that our integrated system provides. The scenario was developed with the help of experienced professional intelligence analysts, and an overview of the storyline is shown in Figure 4. Two analysts, Ella and Joe, are engaged in the investigation of suspicious events in the fictional region of Kish. The first phase features a bacteriological outbreak in Kish, monitored by field patrols over a long period of time where analysts reflect on causes and possible future implications. The second phase starts with a report of an hotel explosion. Here, analysts focus on the timely elaboration of information to map the evolution of events, to provide immediate response to the population affected and to identify potential causes and associations. Ella and Joe collaborate via CISpaces and actively interact 


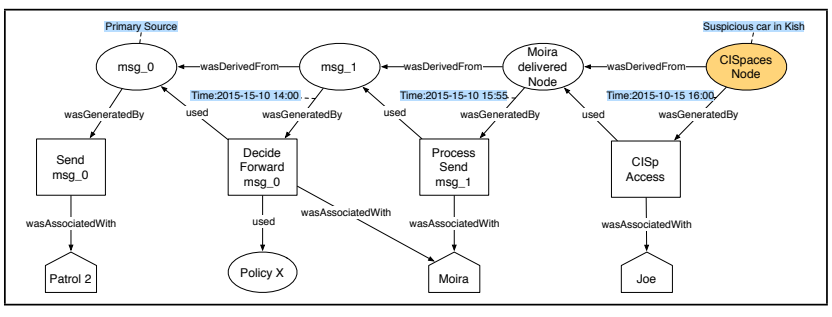

Figure 5: Step 1 - PROV-DM model of Moira node

through Moira with collaborators patrolling the region.

Step 1: Phase 1 - Sensing. Patrol 1, operating in the region, indicates an unidentified illness affecting the local population and their livestock in rural areas of Kish. Patrol 2 observes a black saloon car in the region. Both patrols initiate a conversation with Moira and report their findings. Patrol 2 may report "Black saloon car sighted at Kish Square". Moira interprets the natural language input and link it to some previous knowledge, deriving that this is a suspicious car. Following the sharing policies, this information is sent by Moira to Joe, who is responsible for analysing the situation in Kish, issuing the CE tell card:

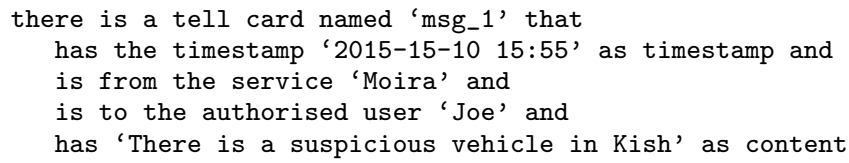

Each card content received by CISpaces is shown as a node in the InfoBox of Joe's space of analysis. Provenance of the information, including the exchange of messages among patrols, Moira and analysts is recorded and shared between systems (see Fig. 5). This step connects sensing to sensemaking between our systems via the CE messaging protocol.

Step 2: Phase 1 - Sensemaking. Joe focuses on the reasons for, and implications of the bacteriological outbreak and identifies the contamination of drinking water as a possible cause of the spread of the illness. The information about the suspicious vehicle seems irrelevant at this point in time. Joe constructs a map of arguments in his space of analysis, where two alternative reasons for the illness are identified: waterborne-bacteria or engineered non-waterborne bacteria contaminate the water supply. The analysis is structured using argument schemes that relate causes of events to effects and opinions from experts (e.g. water tests from the lab). The evaluation of the evidential reasoning agent presents two plausible hypotheses: waterborne bacteria have grown in the water due to a sewage leak and caused the illness to spread across people; alternatively, non-waterborne bacteria were released in the water and caused both illness of people and livestock. The analysis is shown in Figure 6 .

Step 3: Phase 2 - Sensing \& Collaboration. While developing awareness of the situation, analysts receive a notification from Moira of an explosion at a local hotel. The other analyst, Ella is now involved in the crisis action team to deal with the population affected by the explosion. The need for an immediate response requires refocusing of analyst effort, including close collaboration amongst them, and the use of the Moira human/agent services to respond in an informed and timely manner to the situation.

Step 4: Phase 2 - Sensemaking \& Collaboration. Based on his previous analysis, Joe attributes the explo-

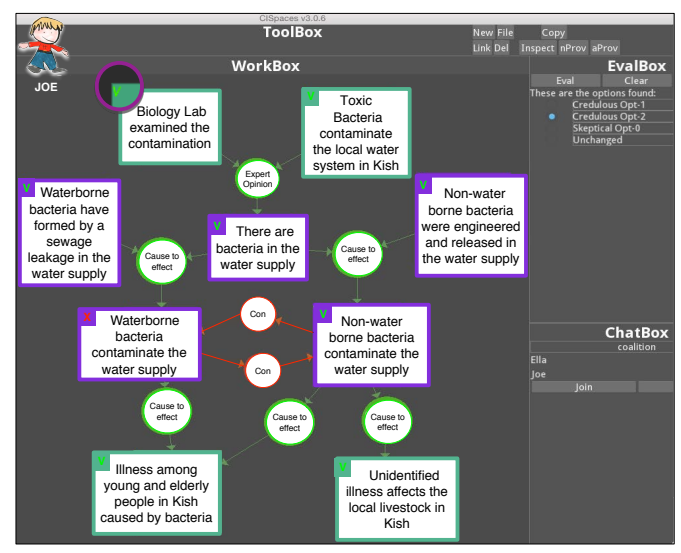

Figure 6: Step 2 - Joe's initial analysis

sion to a gas released in the water supply by the engineered bacteria. Joe shares with Ella his explanation via the CISpaces shared WorkBox, sending the related analysis, which enables Ella to understand and support Joe's reasoning process (Fig. 7). Joe selects a CQ among those proposed by the evidential reasoning agent to request further explanations.

Step 5: Phase 2 - Foraging \& Sensemaking. Ella works on an alternative explanation derived from a different set of information. A suspicious person was seen leaving the hotel and may have planted an explosive. To confirm this hypothesis, Ella initiates a conversation with Moira and requests further information about the person of interest (POI) seen leaving the hotel. CISpaces transforms Ella's message "Who is the POI?" in the card:

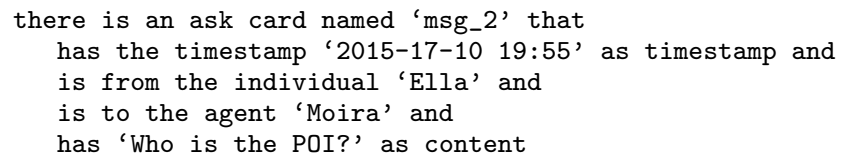

Moira now initiates a BOLO to identify the suspicious person. Reports from the field patrols, and some elaboration of contextual information and previous knowledge from the forensic agents indicate that the POI is a person named John associated to a gang $\mathrm{G}$ operating in the region. This piece of analysis based on direct observation of events at the scene is sent to Ella by Moira as reply to the conversation with a tell card. The new intelligence is structured by Moira as a set of linked Pro nodes returned with the response, permitting a simple and effective introduction of the human reported and agent elaborated information in Ella's space of analysis.

This shows the potential of our integrated system to enable human/agent collaboration from human sensemaking, agent-driven foraging for information, to machine elaboration via forensic reasoning and back to human sensemaking.

Step 6: Phase 2 - Hypotheses Identification. The new hypothesis is now added to the shared space by Ella resulting in two plausible explanations for the hotel explosion: gas released from the water supply, or planted explosive. In order to make a decision, Ella and Joe may be helped by the provenance agent that imports relevant provenance information. Timeliness can be used as criterion to state that the explosive is more preferred due to the fact that the received intelligence from Moira is more recent (see Fig. 7). The additional preference supplied by the provenance agent 


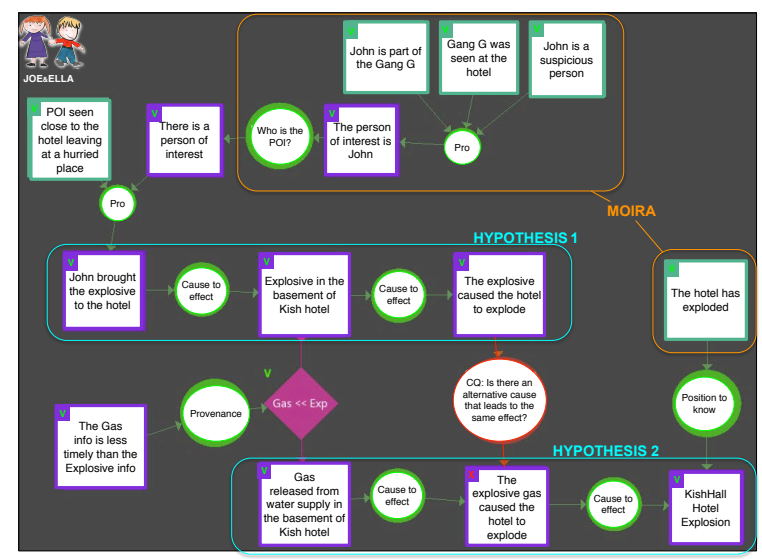

Figure 7: Step 5-6 - Shared environment

will ensure that the evaluation shows only one candidate explanation. This preference may be challenged by CQs, such as whether there are any other criteria that lead to discordant preferences, or whether there is any further evidence for the gas explanation. The use of argumentation permits analysts to reconsider options temporarily deemed as unsupported in the light of new information or further critiques to the current analysis since nodes are never discarded.

Finally, Joe brings back the shared analysis in his individual space. A clearer picture of the situation is now forming: Kish is the focus of a large plan of attacks from gang $\mathrm{G}$. The sighted suspicious vehicle may belong to the gang, which is considered responsible for releasing the engineered bacteria in the water supply and planting the explosive at the hotel.

\section{DISCUSSION AND CONCLUSION}

In this paper, we have explored how the capabilities of CISpaces and Moira complement each other to offer support in reporting and acquiring intelligence and in making sense of this intelligence to identify plausible hypotheses for a situation. Our integrated system uses a variety of state-ofthe-art techniques, such as Controlled Natural Language, argumentation and provenance to enable information foraging and sensemaking, while supporting and interpreting both human and agent contributions. We have demonstrated this process with a detailed walkthrough developed with experienced analysts to show the support offered by Moira and CISpaces to an intelligence coalition while gathering information about, and responding to the attack on Kish.

Further research will focus on strengthening the links between the two systems and evaluating its benefits. We will investigate methods to query and import information into CISpaces taking into consideration different types of data analysis, as well as methods for analysts to be able to send relevant evidence to some of the analysis that the Moira services may perform. The integration of CISpaces and Moira and the scenario discussed in this paper have been developed with the help of professional analysts that will support more thorough evaluation of the system in future work.

While the main application of Moira and CISpaces is intelligence analysis, the aim is to ease the effort of acquiring, evaluating, and interpreting information to better handling evolving complex situations. We believe that our system will provide useful insights into tools to support social sensing and sensemaking of social network information.

\section{Acknowledgments}

This research was sponsored by the U.S. Army Research Laboratory and the U.K. Ministry of Defence and was accomplished under Agreement Number W911NF-06-3-0001. The views and conclusions contained in this document are those of the author(s) and should not be interpreted as representing the official policies, either expressed or implied, of the U.S. Army Research Laboratory, the U.S. Government, the U.K. Ministry of Defence or the U.K. Government. The U.S. and U.K. Governments are authorized to reproduce and distribute reprints for Government purposes notwithstanding any copyright notation hereon.

\section{REFERENCES}

[1] J. A. Burke, D. Estrin, M. Hansen, et al. Participatory sensing. In Proceedings of the ACM Sensys World Sensor Web Workshop, 2006.

[2] M. R. Endsley. Toward a theory of situation awareness in dynamic systems. Human Factors: The Journal of the Human Factors and Ergonomics Society, 37(1):32-64, 1995.

[3] R. J. Heuer. Psychology of intelligence analysis. US Government Printing Office, 1999.

[4] L. Moreau and P. Missier. PROV-DM: The PROV Data Model, April 2013.

[5] D. Mott. Summary of controlled english. Technical report, International Technology Alliance, May 2010. Available at https://www.usukita.org/node/1411.

[6] N. J. Pioch and J. O. Everett. POLESTAR: collaborative knowledge management and sensemaking tools for intelligence analysts. In Proceedings of the 15th International Conference on Information and Knowledge Management, pages 513-521, 2006.

[7] P. Pirolli and S. Card. The sensemaking process and leverage points for analyst technology as identified through cognitive task analysis. In Proceedings of the Int. Conference on Intelligence Analysis, 2005.

[8] A. Preece, D. Braines, D. Pizzocaro, and C. Parizas. Human-machine conversations to support multi-agency missions. ACM SIGMOBILE Mobile Computing and Comm. Review, 18(1):75-84, 2014.

[9] A. Preece, C. Gwilliams, C. Parizas, et al. Conversational sensing. In Proceedings of SPIE, Next-Generation Analyst II, 2014.

[10] A. Preece, W. Webberley, and D. Braines. Conversational sensemaking. In Proceedings of SPIE, Sensing Technology+ Applications, 2015.

[11] A. Toniolo, F. Cerutti, N. Oren, et al. Making informed decisions with provenance and argumentation schemes. In Proc. of the 11th Int. Workshop on Argumentation in Multi-Agent Systems, 2014.

[12] A. Toniolo, T. J. Norman, A. Etuk, et al. Supporting reasoning with different types of evidence in intelligence analysis. In Proceedings of the 14 th International Conference on Autonomous Agents and Multiagent Systems, 2015.

[13] D. Walton, C. Reed, and F. Macagno. Argumentation schemes. Cambridge University Press, 2008. 